\title{
LAS COMPUTADORAS EN LA EDUCACION PRIMARIA: UN ESTUDIO PILOTO ${ }^{1}$
}

\author{
Por \\ Jesús Francisco Galaz Fontes*
}

\section{RESUMEN}

De un grupo de cuarto affo de primaria se seleccionó a diez estudiantes para tomar un pequeño curso de Logo con una maestra, también de primaria, que había sido previamente entrenada en dicho lenguaje de computación. Ambos grupos de sujetos resolvieron un pretest y un postest de aritmética. Los datos muestran que el grupo que recibió el entrenamiento Logo tuvo una mejor ejecución en el postest que el grupo control. Además, las opiniones de los estudiantes, padres de familia y maestra fueron, en general, positivas respecto a la experiencia de aprender a programar Logo, así como en relación al uso de las computadoras en la educación primaria. Se discute si el uso educativo de las computadoras en general, y del lenguaje Logo en particular, puede representar un medio para mejorar el desempeño académico a nivel primaria, sobre todo en zonas urbanas de la frontera norte de México, donde la cultura y las condiciones económicas hacen más viable esta alternativa educativa.

\section{ABSTRACT}

A group of ten students was selected from the fourth grade of elementary school to take a Logo workshop from an elementary teacher previously trained in computer language. Subjects in both groups, experimental and control, took an arithmetic test and a postest. Data collected shows that the group which received

* Psicólogo con maestría de la Universidad de Guelph en Canadá. Es Director del Centro de Desarrollo Integral del Niño. En la actualidad investiga la psicología del desarrollo moral entre la población mexicana y mexicoamericana. Recientemente fue nombrado Profesor Titular en la Escuela de Pedagogía de la Universidad Autónoma de Baja California.

A psychologist with a Masters Degree from tha University of Guelph in Canada. He is Director of the Child Development Center (Centro para el Desarrollo Integral del Niño). Presently he is researching the psychology of moral development among Mexican and Mexican Americans. Currently he is Associate Professor at the School Education of the Autonomous University of Baja California.

${ }^{1}$ El estudio aquí reportado fue posible gracias al apoyo de la IBM Worlwide Education, Entry Systems Division, y de la Secretaría de Educación y Bienestar Social del Estado de Baja California. No obstante, las opiniones vertidas aquí no representan necesariamente su punto de vista. Está basado en un manuscrito leído en el Tercer Simposio Internacional "La computación y la educación infantil y juvenil". Puebla, Puebla, México, octubre 25-31 de 1986. 
instruction in Logo obtained highest scores in the postest than the control group. Furthhermore, students, parents, and the elementary teacher reported positive opinions about their experience or learning Logo programming, and using computers in elementary school. It is concluded that using computers for instrumental purpose in general, and using Logo in particular, could represent a tool to improve academic performance at elementary level, particularly in urban areas along the Mexican northem border where cultural and economic conditions provide a receptive environment for this educational alternative.

\section{LAS COMPUTADORAS EN LA EDUCACION PRIMARIA: UN ESTUDIO PILOTO}

Toda frontera ofrece a sus habitantes un contexto diferente al que encuentran los pobladores del interior de los estados vecinos. Lejos de verse como una situación anómala, el contacto con otra cultura es un factor que debe considerarse como propio del ambiente fronterizo, especialmente en el caso de los niños (Nolasco y Acevedo, 1985). La frontera norte de México, y en especial sus principales centros urbanos (Tijuana, Mexicali, Ciudad Juárez), ofrecen a su población un ambiente cultural que posibilita, sobre todo en los niveles socioeconómicos medio y alto, conocer los últimos desarrollos tecnológicos. La computadora es uno de éstos y, como en otros casos (videocaseteras, por ejemplo), a pesar de que podría usarse positivamente en el ámbito educativo, hasta hoy ha permanecido alejada, sobre todo de la educación básica, predominando los usos comerciales de la misma.

Mexicali colinda con California y, en parte debido a ello, ofrece un ambiente en donde el contacto con las computadoras es cada vez más frecuente. Los comercios, las fábricas, los bancos y el propio gobierno usan cada vez más intensamente las computadoras, así como las instituciones locales de educación superior. Por lo tanto, la idea de incorporar la informática al ámbito educativo básico está lejos de ser descontextualizada. Además, como en esta ciudad existen facilidades para la obtención de equipos y programas, la viabilidad de usar las computadoras dentro de las escuelas primarias no tiene por qué verse limitada a las escuelas particulares que tienen los recursos y la inquietud por explorar los usos educativos de esta tecnología. Dentro de este contexto, el Centro para la Educación Integral del Niño presentó algunas iniciativas en este sentido a la Secretaría de Educación y Bienestar Social de Baja California; como un primer paso se decidió realizar un estudio piloto con la finalidad de evaluar el impacto del uso de la computadora como apoyo en el programa de matemáticas de cuarto año de primaria (Anónimo, 1987, Galaz Fontes, 1986, 1987). Habiéndose suspendido el proyecto global por cuestiones ajenas al desarrollo del mismo, hemos juzgado pertinente rescatar parte del estudio piloto mencionado y reportarlo aquí. Así pues, el presente estudio representa una exploración en cuanto al uso educativo de la computadora en el contexto fronterizo de Mexicali. 
Durante las tres últimas décadas, los países desarrollados del mundo han sido testigos de cómo la computadora ha impactado todos los aspectos de la vida humana (Sanders, 1985). Aunque en un principio solamente usada en granden proyectos educativos como piloto (Curran y Curnow, 1984), la aparición del microprocesador abrió, en dichos países, la posibilidad de su uso masivo en ln educación. Así, las computadoras se usan cada día con mayor frecuencia, como tutores individuales y como auxiliares didácticos, como herramientas para ol trabajo académico e intelectual, y como una materia acerca de la cual es con. veniente aprender (Clements, 1985). Por otro lado, su uso representa, en tales países, un lugar común tanto en los niveles educativos superiores como en los elementales ( $V . g r$. Hess \& Ford, 1985; Mehan, Moll \& Riel, 1985).

Las computadoras, como otras tecnologías, han sido desarrolladas y más ampliamente usadas en los países desarrollados. Los países en vías de desarrollo, y en especial los que no hablan inglés, no las han utilizado tanto, ni siquiera en los niveles superiores de su educación. En México, sin embargo, tal situación ha cambiado significativamente en la última década. En un principio usada preponderantemente dentro de la administración de la educación superior (v.gr. Fok Pun, 1985), la computadora ha empezado a ser motivo de reflexión en el área de la educación infantil (v.gr. Academia de la Investigación Científica, 1985, 1986), en donde ya se habla de que "es urgente iniciar trabajos de introducción de computadoras en la educación primaria y si fuera posible en preescolar. Simultáneamente debe hacerse una intensa labor de sensibilización entre todos los maestros del sistema educativo nacional" (Bustamante, 1986: 15). Ante esta visión de que la computadora representa la promesa de una "gran revolución educativa" (Calderón Alzati, 1986), se ha comentado también la posibilidad de que su uso inadecuado pueda generar consecuencias negativas (Herrera, 1986), lo cual nos hace ver la necesidad de mantener una actitud evaluativa (Weiss, 1975) al incorporar la computadora a la educación básica. En particular, es conveniente que en México pongamos atención en minimizar la discriminación socioeconómica y sexual, en cuanto al uso educativo de las computadoras, que se sabe ha acontecido en países como Estados Unidos (DeVillar, 1986; DeVillar y Faltir, 1987).

Como se deja vislumbrar en el párrafo anterior, un factor esencial para la integración de la computadora a la educación será el elemento humano. Respecto a la escuela, el maestro es la persona que pone en práctica cualquier reforma educativa; él es el experto en el nivel operativo y, por lo tanto, es natural que juegue un rol central en cuanto al éxito o fracaso de cualquier iniciativa de innovación educativa. En particular, si las computadoras han de tener un impacto positivo a gran escala, el maestro tendrá que participar significativamente en tal esfuerzo.

Teniendo tal importancia, resulta paradojico que en México el nivel profesional del maestro sea considerado generalmente bajo. Seguramente que esto ha sido un factor para que algunos proyectos sobre computación infantil trabajen directamente con los niños a través de instructores especializados, como en el caso de 
lus Centros Galileo (Leyva, 1986), no fomentando así el contacto del maestro de escuela con estos avances tecnológicos. En contraste con esta situación, nosotros pensamos que resulta imperativo trabajar con maestros, tanto en servicio como en formación; ambos forman parte de la realidad de nuestro sistema educativo y, si pretendemos afectarlo, necesitamos motivar su participación. En este sentido, nuestro estudio investigó la viabilidad de que sean los maestros los que trabajen directamente con sus estudiantes.

No obstante, e independientemente de lo anterior, al final de cuentas el uso de las computadoras como auxiliares didácticos se justificará en la medida en que contribuya a mejorar el desempeño académico de los estudiantes. En este sentido, y dado lo importante y lo problemático del área de las matemáticas, se eligió a la misma comoun primer punto factible de trabajarse con la ayuda de la computadora; considerando, por otro lado, el reconocido valor en esta área de Logo(v. gr. Dale, 1986; Grillo A., 1985; Papert, 1980), un lenguaje estructurado de programación basado en gráficas y "comandos" (instrucciones) sumamente "amigables" para que se realicen dibujos sobre la pantalla de la computadora. Nuestro estudio se planteó la interrogante de si el aprender Logo con la ayuda de una maestra de primaria mejoraría el desempeño en matemáticas de estudiantes de cuarto año de una escuela de nivel socioeconómico medio-bajo en la ciudad fronteriza de Mexicali.

\section{METODO}

\section{Sujetos}

Una maestra de primaria (mujer de 36 años de edad, con 15 de experiencia docente, a cargo de un primer año, y sin ninguna experiencia con computadoras) se prestó a trabajar voluntariamente en el proyecto como instructora de un grupo de Logo o experimental de 10 estudiantes (7 hombres y 3 mujeres), con una edad promedio de 9.6 años. Tales sujetos, junto con los 24 del grupo control ( 10 hombres y 14 mujeres; edad promedio de 9.9 años), formaban una clase de 34 niños del cuarto año de primaria de una escuela localizada en un área socioeconómica mediabaja de la ciudad de Mexicali.

\section{Aparatos e instrumentos}

El salón de computadoras del Centro para la Educación Integral del niño (CEIN), una institución educativa privada situada en un área socioeconómica media de la ciudad, sirví́ como escenario tanto del entrenamiento de la maestra, como del taller de programación para los estudiantes del grupo de Logo. En dicho espacio se contó con cinco computadoras IBM PCjr con $256 \mathrm{~KB}$ de memoria y monitor a color, las cuales además tenían un impresor cada una. Se utilizó una traducción en español de la versión IBM del lenguaje Logo (Logo Computers Systems, 1983). Sin embargo, tal traducción se modificó localmente, ya que se 
consideró que utilizar términos familiares facilitaría las primeras interacciones do la maestra y los niños con el programa.

\section{Procedimiento}

En primer lugar, la maestra de primaria recibio capacitación del responsable dol proyecto sobre el uso del lenguaje Logo. Tal capacitación tuvo tres objetivon: permitir que la maestra tuviera un primer contacto con los usos educativos de la computadora, familiarizarla con el lenguaje Logo y, finalmente, que la maestra elaborara un programa que integrara actividades de programación Logo con objetivos específicos del programa de matemáticas de cuarto año.

La capacitación de la maestra se realizó en poco más de un mes (19 sesiones do trabajo, 41 horas de capacitación). Después de una breve introducción al tema dol uso educativo de las computadoras, se describió en qué consiste una computadorı y, en particular, se revisaron los componentes del sistema PCjr. Luego de ello se revisaron 35 "comandos", operaciones y elementos del lenguaje Logo. El capacitador demostraba el comportamiento de tales elementos y, enseguida, se sugerfa a la maestra posibles proyectos (dibujos) a realizar con base en ellos. Al mismo tiempo, se fomento que extrajera actividades concretas del programa do matemáticas, lo cual pronto brindo el contexto para practicar los "comandos" revisados, así como el incentivo para introducir otros conforme fueron necesarios. Por último, la maestra elaboró un plan de trabajo para los estudiantes de cuarto grado, así como dos pruebas equivalentes de matemáticas para aplicarse, al inicio y término del taller, a todos los niños involucrados en el estudio. Las pruebas se basaron en los objetivos del programa oficial de matemáticas para el cuarto año do primaria (Secretaría de Educación Pública, 1984), y versaron sobre ejes de simetría, ángulos, dibujos a escala, áreas y figuras geométricas.

Luego de la capacitación de la maestra, ella misma fungió como instructora do los 10 estudiantes del grupo Logo. A pesar de que se recomendó que la selección de los niños se hiciera al azar, el grupo Logo presentó calificaciones escolares do matemáticaas más altas que el grupo control (8.3 vs. $7.33, \mathrm{t}=2.35, \mathrm{p}<.0269$. Ello obligó a que en el análisis de los resultados se tomara en cuenta la calificación de matemáticas como una covariable, de modo que se pudiera asegurar que las diferencias observadas estuvieran asociadas al tratamiento experimental, y no a las diferencias encontradas inicialmente en el desempeño académico.

El grupo Logo asistió al CEIN, después de sus clases matutinas normales, dos veces por semana durante mes y medio (15 sesiones, 28 horas de trabajo) y, en promedio, cada sujeto asistió a $90 \%$ de las sesiones. A lo largo del taller se enfatizo el trabajo en equipo, ya que dos estudiantes -no siempre necesariamente los mismos- estuvieron trabajando con cada computadora. En general, la maestra primero presentaba un nuevo "comando" a todo el grupo, señalando qué hacer 
sobre el teclado y apuntando qué observar en el monitor. Después de ejemplificar su funcionamiento, se requerían proyectos específicos de parte de los niños (dibujar un cuadrado en la esquina superior derecha de la pantalla, dibujar las líneas de simetría de una figura, etc.). Por último, también se fomentó la creatividad de los estudiantes estimulándolos a que realizaran proyectos propios. Durante todo el taller la maestra y el autor se reunieron una vez por semana para discutir el avance del mismo.

A ambos grupos se les aplicó el pre y el postest de matemáticas anteriormente referido. Además, el grupo Logo fue evaluado por el responsable del proyecto en relación con los contenidos del taller y, al final de éste, los estudiantes y la maestra contestaron un cuestionario de opinión respecto al mismo. Al término del taller, también se mandó un cuestionario sobre el uso educativo de la computadora a los padres de todos los niños involucrados en la experiencia. El cuestionario incluyó una serie de preguntas tipo Likert en relación con los posibles efectos educativo y social de la computadora.

\section{RESULTADOS}

Respecto a las evaluaciones del contenido del taller, el promedio del grupo Logo fue, en una escala de 10, de $7.4(S x=13.3)$, por lo que puede afirmarse que los niños lograron aprender los principios básicos de la programación Logo. Por otro lado, y con base en los cuestionarios que los estudiantes del grupo Logo contestaron al término del taller, resulta evidente que esta experiencia les agradó mucho. Trabajar en parejas fue motivante y, de una manera uniforme, todos los niños del grupo Logo consideraron que el taller había afectado positivamente su trabajo y, en particular, su desempeño en matemáticas. Finalmente, todos los estudiantes estuvieron satisfechos del trabajo de su instructora y manifestaron estar dispuestos a asistir en un futuro a talleres semejantes.

En relación con el impacto académico del taller, se realizó un análisis de covarianza (Crunch Software Corporation, 1986) para eliminar la variabilidad en las calificaciones del pretest y postest, que pudieran asociarse con la diferencia inicial en calificaciones de matemáticas entre los grupos Logo y Control. El análisis de covarianza fue de un factor grupo entre sujetos (grupo Logo vs. grupo Control) y un factor prueba intrasujetos (pretest vs. postest). Dado que se esperaba que el grupo Logo aventajara al grupo Control sólo después de haber participado en el taller de programación Logo; nuestra hipótesis de trabajo implicaba que el análisis de covarianza sólo arrojaría un efecto significativo en la interacción grupo $x$ prueba. Como se puede observar en el cuadro 1 y como también era de esperarse, el efecto de la covariable calificación de matemáticas fue significativo $\left(\mathrm{F}_{1}{ }_{31}=25.511, \mathrm{p}<.00000\right)$, confirmando así que una parte significativa de los resultados podía atribuirse al desempeño diferencial en matemáticas al inicio del estudio. Sin embargo, el efecto del factor grupo no fue significativo $\left(F_{1,31}=0.123\right.$, 
$\mathrm{p}<.728)$, lo mismo que el efecto del factor prueba $\left(F_{1,32}=1.621, \mathrm{p}<.2121\right)$. No obstante, la interacción de los factores grupo y prueba fue significativa $\left(\mathrm{F}_{1,32}=7.468, \mathrm{p}<.0101\right)$. Dicha interacción fue debida, tal como se había prevista, al incremento, relativo al pretest, del postest del grupo Logo (medias de $6.59 \mathrm{vs}$. 8.06, respectivamente; $t_{9}=3.748, p<.0046$; ver figura 1).

En cuanto al cuestionario sobre el uso educativo de la computadora, se observó que tanto los padres del grupo Logo, como los de grupo Control, tenían una mínima experiencia con las computadoras. No obstante, ambos grupos de padres manifestaron una actitud positiva respecto a las implicaciones educativas, laborales y sociales del uso educativo de la computadora. En esos mismos cuestionarios se les pregunt 6 a los padres de familia acerca del nivel educativo en el que ellos pensaban que los estudiantes deberían empezar a trabajar con computadoras. Noventa por ciento de los padres del grupo Logo contestó que antes de terminar la primaria, mientras que en el grupo control el porcentaje correspondiente fue del $35 \%$. Tal diferencia, de acuerdo con la prueba exacta de Ficher (Siegal, 1970), es significativa al nivel de .01 . Es muy probable que el ver a sus hijos participar exitosamente en esta experiencia haya afectado la opinión de los padres del grupo Logo.

Por último, se hicieron cinco preguntas a los padres del grupo Logo respecto ॥ la participación de sus hijos en el taller. Todos los padres respondieron haber platicado con sus hijos acerca del taller, y todos vieronla experiencia como positiva en términos de mejores hábitos de pensamiento, reforzamiento del gusto por la escuela, y otros aspectos. Por otro lado, ninguno de ellos comunicó algún efecto colateral negativo en relación al desempeño escolar de sus hijos. Finalmente, todos los padres manifestaron su apoyo en relación a futuras actividades educativas que tengan que ver con computadoras.

La maestra también resolvió un cuestionario respecto a su experiencia, el cual indicó un alto entusiasmo respecto al uso educativo de las computadoras. Asimismo, manifestó estar dispuesta a participar en futuros talleres. La maestra reconocí́ el potencial existente en esta tecnología para mejorar el pensamiento geométrico y matemático de los niños, aunque también mostró estar consciente de las dificultades implicadas en un uso masivo de la computadora (aspectos económicos y de capacitación, sobre todo). La maestra también manifestó que hubiese sido conveniente más tiempo tanto para su capacitación, como para el trabajo directo con los niños.

\section{DISCUSION}

Como se puede observar por el nivel de asistencia al taller de programación, los estudiantes participantes y sus padres hicieron un esfuerzo para beneficiarse de esta experiencia. Además, desde una perspectiva social y educativa general, podemos afirmar que los niños participantes, de condiciones socioeconómicas 
media-baja, se mostraron muy capaces y entusiastas de aprender a usar una computadora. A pesar de que los sujetos sólo trabajaron 28 horas aprendiendo a programar Logo, y por lo tanto es posible que el entusiasmo mostrado sea un efecto de novedad, este estudio al menos sugiere que los niños de cuarto año de primaria tumbién se pueden beneficiar del trabajo con computadoras. Seguramente que es algo fundamental que esta tecnología, y en especial el lenguaje Logo con sus actividades de dibujo, puede combinar exitosamente el aprendizaje y el interes.

Observaciones informales nos muestran que las computadoras son cada día más comunes en el ámbito general de Mexicali, y seguramente que ello influyb en la disposición y actitud de los estudiantes para aprender Logo, además de la natural actitud exploratoria de los niños. Por otro lado, tanto los maestros como los niños del grupo Logo trabajaron con una traducción en español. Además del atractivo gráfico, es muy probable que este factor haya sido medular para la aceptación y entusiasmo mostrado por todos los participantes ya que no tuvieron necesidad de invertir ningún esfuerzo en traducir, ni lo que querían "decirle" al programa, ni los mensajes que el programa a su vez les mandaba.

Los estudiantes del grupo Logo mostraron un aumento significativo en un postest de matemáticas en relación al grupo Control con el que habían sido comparables en un pretest. A pesar de que no se discutan aquí los mecanismos psicológicos potencialmente responsables de tal efecto, este resultado es importante en tanto muestra que el uso de la computadora en general y, en particular, el uso del lenguaje Logo, puede tener consecuencias educativas concretas. El carácter tentativo de esta conclusión se debe a que los grupos Logo y Control difirieron entre sí en aspectos que bien pudieron haber colaborado en la determinación de los resultados encontrados. Entre tales aspectos cabe señalar el hecho de que el grupo Logo recibí una enseñanza adicional en matemáticas, sus integrantes trabajaron en parejas y su maestra recibi 6 una asesoría semanal durante el taller. Se necesita un tipo de investigación más analítica para poder desentrañar la contribución relativa de cada uno de estos factores a los resultados aquí reportados.

No obstante estas salvedades y considerando que el taller durb 28 horas, uno bien se podría preguntar qué pasaría si una experiencia similar se programara a lo largo de todo el ciclo escolar. Matemáticas es una de las áreas más difíciles para los estudiantes, pero también es un área esencial para obtener una formación más sólida. Logo bien podría ser parte de la respuesta en cuanto a hacer de la geometría y de las matemáticas algo más concreto, obvio y agradable para los niños. Dadas las necesidades de profesionistas en las disciplinas tecnológicas, resulta pertinente continuar explorando las posibilidades que se deriven del presente estudio.

Como se podrá haber observado, la maestra elaboró el programa para integrar las actividades Logo con objetivos específicos del programa de matemáticas, y ella 
fungió como instructora durante el taller. Dada la capacitación que los maestros han adquirido a lo largo de su formación, y considerando su experiencia docente, vemos que toda esta participación fue esencial para el buen éxito del proyecto. Así pues, si las computadoras han de tener un impacto educativo significativo a gran escala, las debemos integrar a las escuelas, y al hacer esto las estaremos poniendo, en última instancia, en las manos de los profesores. La experiencia adquirida en este estudio muestra que hacer esto es posible. En este aspecto, un siguiente paso sería trabajar con grupos de maestros, de manera que empecemos a investigar las condiciones para generalizar a partir de los resultados aquí reportados. En general, muchos de los nifios de hoy en día encuentran que la escuela es aburrida, mientras que el mundo exterior es mucho más interesante y atractivo. Si consideramos que las computadoras pueden ser usadas en una gran variedad de actividades académicas y que, por lo tanto, su costo no estaría justificado exclusivamente por un sólo uso, la adquisición de computadoras en el sistema educativo oficial no debe verse como un gasto desorbitado. Introducir las computadoras a la escuela, y no tanto el llevar los nifios a las mismas, puede mejorar la realidad educativa e imagen de la escuela. Asimismo, habría una mayor concordancia con ese mundo de afuera. Tal puede ser la situación de un ambiente fronterizo como en el que se llevó a cabo esta experiencia.

FIGURA 1. Evaluación de matemáticas.

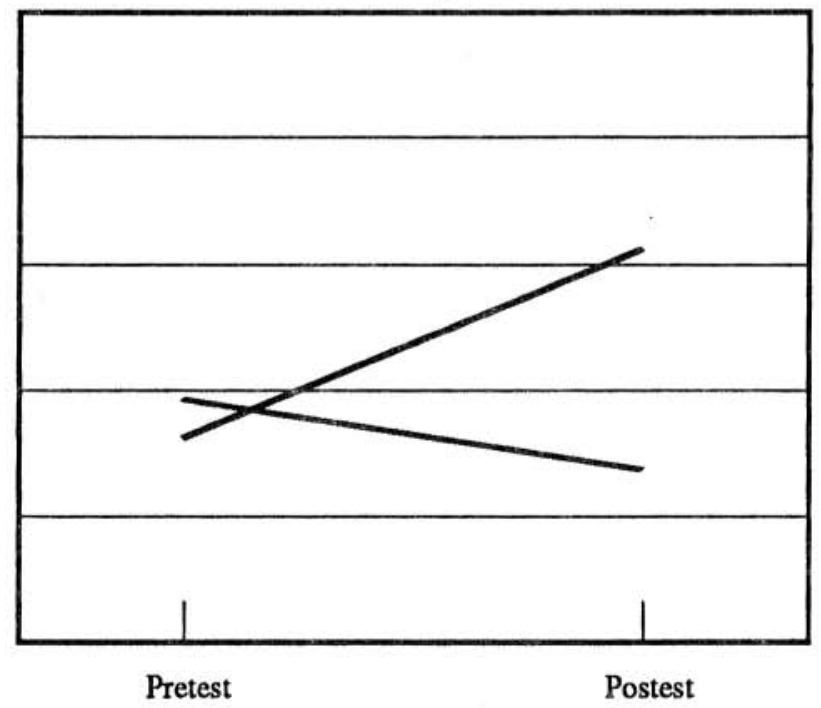

_ Grupo Logo

Grupo Control 
CUADRO 1. Análisis de covarianza de los resultados del estudio.

\begin{tabular}{lllllc}
\hline Análisis de varianza & gl & \multicolumn{1}{c}{ SC } & MSC & F & P \\
Entre sujetos & 33 & 185.1295 & & & \\
Covariables & 1 & 83.3929 & 83.3929 & 25.511 & 0.0000 \\
Calif. Mat. & 1 & 83.3929 & 83.3929 & 25.511 & 0.0000 \\
Grupo & 1 & 0.4026 & 0.4026 & 0.123 & 0.7280 \\
Ss intragrupos & 31 & 101.3340 & 3.2688 & & \\
& & & & & \\
Intra sujetos & 34 & 74.8616 & & & \\
Pr-Ps & 1 & 3.0733 & 3.0733 & 1.621 & 0.2121 \\
Grupo x Pr-Ps & 1 & 14.1554 & 14.1554 & 7.468 & 0.0101 \\
Pr-Ps x Ss intragrupos & 32 & 60.6562 & 1.8955 & & \\
\hline
\end{tabular}

\section{BIBLIOGRAFIA}

ACADEMIA DE LA INVESTIGACION CIENTIFICA (Comp.) 1985.Memoria del Segundo Simposio Internacional "La computación y la educación infantil" . México: Academia de la Investigación Cientifica.

- 1986. Memoria del Tercer Simposio Internacional "La computación y la educación infantil y juvenil". México: Academia de la Investigación Cientifica.

Anónimo 1987. "La computadora en la educación primaria". Pulso educativo (Organo de Difusión de la Secretaría de Educación y Bienestar Social del Gobierno del Estado de Baja California), No. 29, junio 1987.

BUSTAMANTE, J. 1986. "Impulso a la computación infantil". Información cientffica y tecnológica, 8 (113), febrero 1986.

CALDERON Alzati, E. 1986. "La gran revoluión educativa". Información cientifica y tecnologica, 8 (113), febrero 1986.

CLEMENTS, D. H. 1985.Computers in early and primary education. Englewood Cliffs, N. J.: Prentice-Hall.

CURRAN S., R. Curnow, 1984. El estudiante y el ordenador: Aplicaciones a la enseñanza. Barcelona: Gustavo Gili.

CRUNCH SOFTWARE CORPORATION 1986. Interactive statiscal package (Crisp). San Francisco, Ca.: Crunch Software Corporation.

DALE, E.J. 1986. "Logo builds thinking skills".In: D. O. Harper and J. H. Stewart (Eds.), Run: Computer education (2nd. ed.). Monterey, Calif.: Brooks/Cole.

DEVILLAR, R. A. 1986. Computer and educational equity within the United States: An overview and examination of computers used in education. Working Paper, Stanford-UNESCO Symposium on Computers and Educarion. Stanford University, March 10-14. 


\section{LAS COMPUTADORAS EN LA EDUCACION PRIMARIA}

FOK, Pun, L. 1985. "El uso de microcomputadoras en control escolar". En: Fundación Arturo Rosenblueth (Comp.), Memoria de la V Conferencia en la Educación" (pp. ii/2-ii/9). México: Fundación Arturo Rosenblueth.

GALAZ Fontes, J. F. 1986. "El uso de la computadora en la educación básica". Pulso educativo (Organo de difusión de la Secretaría de Educación y Bienestar Social del Gobiemo del Estado de Baja California), No. 18, junio 1986.

- 1987. "La computadora en la educación primaria". Pulso educativo (Organo de difusión de la Secretaría de Educación y Bienestar Social del Gobierno del Estado de Baja California), No. 30, julio 1987.

GRILLO A., 1985. La enseñanza de Logo y su integración en la escuela primaria. En: Academia de la Investigación Científica (Comp.),Memoria del Segundo Simposio Internacional "La computación y la educación infantil" (pp. 12.112.7). México: Academia de la Investigación Científica.

HERRERA, N. 1986. "Aprendizaje o enajenación". Información científica y tecnológica, 8 (113), febrero 1986.

HESS, R. D. \& M. E. Ford, 1985. Effects of microcomputer use in kindergarten on students social behavior and academic performance. Stanford, Ca.: Stanford University. December 1985.

LEYVA, J. A. 1986. "Enseñanza del futuro: La visión de Galileo". Información cientifica y tecnologica, 8 (113), febrero 1986.

LOGO COMPUTER SYSTEM 1983. Logo. Boca raton, Florida: IBM.

MEHAN, H., L., Moll, and Riel, M.M.(Eds.) 1985 Computers in classrooms: A quasiexperiment in guided change (final report). La Jolla, Ca.: University of California, San Diego. June 26, 1985.

NOLASCO, M., y M.L. Acevedo, 1985.Los niños de la frontera: ¿Espejismos de una nueva generación? México, Océano y Centro de Ecodesarrollo.

PAPERT, S. 1980. Mindstorms. New York: Basic Books.

SANDERS, D.H. 1985. Informática: Presente y futuro. México: McGraw-Hill. SECRETARIA DE EDUCACION PUBLICA. 1984. Libro para el maestro, 40. grado (3ra. ed.). México: Secretaría de Educación Pública.

SIEGEL, S. 1956/1970. Estadística no paramétrica. México: Trillas.

WEISS, C.H. 1975. Investigación evaluativa. México: Trillas. 